\title{
Photoionization of ground and excited levels of P II
}

\author{
Sultana N. Nahar \\ Department of Astronomy, The Ohio State University, Columbus, OH 43210 \\ Email: nahar.1@osu.edu, tel: 1-614-292-1888, fax:1-614-292-2928
}

\begin{abstract}
Photoionization cross section $\left(\sigma_{P I}\right)$ of $\mathrm{P}$ II, $(\mathrm{h} \nu+\mathrm{P}$ II $\rightarrow \mathrm{P}$ III $+\mathrm{e})$, from ground and a large number of excited levels are presented. The study includes the resonant structures and the characteristics of the background in photoionization cross sections. The present calculations were carried out in the Breit-Pauli R-matrix (BPRM) method that includes relativistic effects. The autoionizing resonances are delineated with a fine energy mesh to observe the fine structure effects. A singular resonance, formed by the coupling of channels in fine structure but not allowed in LS coupling, is seen at the ionization threshold of photoionization for the ground and many excited levels. The background cross section is seen enhanced compared to smooth decay for the excited levels. Examples are presented to illustrate the enhanced background cross sections at the energies of the core levels, ${ }^{4} P_{3 / 2}$ and ${ }^{2} D_{3 / 2}$, that are allowed for electric dipole transitions by the core ground level ${ }^{2} P_{1 / 2}^{o}$. In addition strong Seaton or photo-excitation-of-core (PEC) resonances are found in the photoionization of single valence electron excited levels. Calculations used a close coupling wave function expansion that included 18 fine structure levels of core P III from configurations $3 s^{2} 3 p, 3 s 3 p^{2}, 3 s^{2} 3 d, 3 s^{2} 4 s, 3 s^{2} 4 p$ and $3 p^{3}$. Photoionization cross sections are presented for all 475 fine structure levels of P II found with $n \leq 10$ and $l \leq 9$. The present results will provide high precision parameters of various applications involving this less studied ion.
\end{abstract}

Keywords: Photoionization of P II, Breit-Pauli R-matrix method, Autoionizing resonances, Relativistic effects at low energy 32.10-f,95.30.Ky,32.80-t

\section{Introduction}

Phosphorus is part of our daily life in variety of applications. Being an element of RNA where it plays a role in signal passing, it is also a basic element

\footnotetext{
Email addresses: nahar.1@osu.edu (Sultana N. Nahar), Tel:1-614-292-1888, Fax: 1-292-2928 (Sultana N. Nahar)

$U R L:$ http://www.astronomy.ohio-state.edu/ nahar (Sultana N. Nahar)
}

Preprint submitted to New Astronomy

July 1, 2016

(C) 2016. This manuscript version is made available under the Elsevier user license http://www.elsevier.com/open-access/userlicense/1.0/ 
like carbon, oxygen in the evolution of life. However, its astrophysical abundance is low and is difficult to detect due to lack of data. Its existence in astronomical objects has been found in damp Galaxies by Molaro et al. [1] and by Welsh et al. [2]. Compared to other elements, only very limited theoretical study has been carried out on the radiative processes of this element.

Photoionization cross sections of Si-like ions, isoelectronic to P II, have been reported under the OP [3] by Mendoza and Zeippen [4], Nahar and Pradhan [5]. However, $\mathrm{P}$ which is the next element to $\mathrm{Si}$ in the periodic table, was not studied due to lower abundance in astrophysical plasmas. Photoionzation cross section of P II has been measured recently with high precision at the Advanced Light Source (ALS) by Hinojosa et al [6] where they studied the low energy features in detail. The observed features were identified theoretically using R-matrix method and were found to be produced by low lying fine structure levels that belong to ground configuration $3 s^{2} 3 p^{2}$ and one metastable state $3 s 3 p^{3}\left({ }^{5} S_{2}^{o}\right)$. The present work reports full scale study of features of photoionization, such as, background enhancement, photo-excitation-of core resonances, and reporting cross sections of a large number of excited levels, 475 in total with $\mathrm{n} \leq 10$ as needed for spectral modelings. In contrast to the study of photoionization of Si-like ions in non-relativistic LS coupling [5], the present study employed the Breit-Pauli R-matrix (BPRM) method with relativistic effects developed under the Iron Project [7] and includes radiation damping effect of resonances [8].

\section{Theory}

Photoionization process can be direct as,

$$
h \nu+X^{+} \rightarrow e+X^{2+} .
$$

However, depending on the photon energy which matches the energy of a state belonging to Rydberg series of states below an excited core state but lying above the ionization threshold, an intermediate doubly excited autoionization state can form before ionization,

$$
h \nu+X^{+} \leftrightharpoons\left(X^{+}\right)^{* *} \leftrightharpoons e+X^{2+}
$$

Formation of an autoionizing state can lead to a resonance in photoionization and is the main cause for introduction of features and structures in the process.

Theoretically the resonances can be generated naturally in an ab initio manner by including the core excitations in the wave function, as considered in the close coupling (CC) approximation. In CC approximation, the atomic system is represented by a $(\mathrm{N}+1)$ number of electrons where the core ion is an $\mathrm{N}$ electrons system interacting with the $(\mathrm{N}+1)$ th electron. The $(\mathrm{N}+1)$ th electron can be bound or in the continuum depending on its negative or positive energy (E). The total wave function, $\Psi_{E}$, in a symmetry $S L \pi$ of the system is expressed by an expansion as (e.g. [9])

$$
\Psi_{E}(e+i o n)=A \sum_{i} \chi_{i}(\text { ion }) \theta_{i}+\sum_{j} c_{j} \Phi_{j}
$$


where $\chi_{i}$ is the core ion eigenfunction representing ground and various excited states and the sum is over the number of core states considered. The core is coupled with the $(\mathrm{N}+1)$ th electron function, $\theta_{i}$. The $(\mathrm{N}+1)$ th electron with kinetic energy $k_{i}^{2}$ is in a channel labeled as $S_{i} L_{i}\left(J_{i}\right) \pi_{i} k_{i}^{2} \ell_{i}[S L(J) \pi] . A$ is the antisymmetrization operator. In the second sum, the $\Phi_{j}$ s are bound channel functions of the $(\mathrm{N}+1)$-electrons system that provides the orthogonality between the continuum and the bound electron orbitals and account for short range correlation. Substitution of $\Psi_{E}(e+i o n)$ in the Schrodinger equation

$$
H_{N+1}^{B P} \Psi_{E}=E \Psi_{E}
$$

introduces a set of coupled equations that are solved using the R-matrix approach. The details of the R-matrix method in the $\mathrm{CC}$ approximation can be found in, e.g. [10, 11, 12, 13, 9]. The relativistic effects are included through Breit-Pauli approximation (e.g. [9]) where the Hamiltonian is given by

$$
\begin{aligned}
H_{N+1}^{\mathrm{BP}}= & \sum_{i=1}^{N+1}\left\{-\nabla_{i}^{2}-\frac{2 Z}{r_{i}}+\sum_{j>i}^{N+1} \frac{2}{r_{i j}}\right\}+ \\
& H_{N+1}^{\mathrm{mass}}+H_{N+1}^{\mathrm{Dar}}+H_{N+1}^{\mathrm{so}},
\end{aligned}
$$

in Rydberg unit. The relativistic correction terms are mass correction, $H^{\text {mass }}=$ $-\frac{\alpha^{2}}{4} \sum_{i} p_{i}^{4}$, Darwin, $H^{\text {Dar }}=\frac{Z \alpha^{2}}{4} \sum_{i} \nabla^{2}\left(\frac{1}{r_{i}}\right)$, and spin-orbit interaction, $H^{\text {so }}=$ $Z \alpha^{2} \sum_{i} \frac{1}{r_{i}^{3}} \mathbf{l}_{\mathbf{i}} \cdot \mathbf{s}_{\mathbf{i}}$. R-matrix Breit-Pauli (BPRM) approximation also includes part of two-body interaction terms, such as the ones without the momentum operators [9]. In BPRM method the set of $S L \pi$ is recoupled for $J \pi$ levels of (e + ion) system in intermediate coupling which is followed by diagonalization of the Hamiltonian.

For an electron with positive energies $(\mathrm{E}>0)$ the solution of the BPRM method is a continuum wave function, $\Psi_{F}$ and with negative energy $(\mathrm{E} \leq 0)$ it is a bound state, $\Psi_{B}$. The complex resonant structures in photoionization are produced from couplings between continuum channels that are open $\left(k_{i}^{2}>\right.$ $0)$, and bound channels that are closed $\left(k_{i}^{2}<0\right)$, at electron energies $k_{i}^{2}$ which correspond to autoionizing states of the Rydberg series, $S_{i} L_{i} J_{i} \pi_{i} \nu \ell$. where $\nu$ is the effective quantum number of the series converging to excited core thresholds $S_{i} L_{i} J_{i} \pi_{i}$.

The photoionization cross section $\left(\sigma_{P I}\right)$ is given by (e.g. [9])

$$
\sigma_{P I}=\frac{4 \pi^{2}}{3 c} \frac{1}{g_{i}} \omega \mathbf{S},
$$

where $g_{i}$ is the statistical weight factor of the bound state, $\omega$ is the incident photon energy and $\mathbf{S}$ is the generalized line strength

$$
\mathbf{S}=\left|<\Psi_{f} \| \mathbf{D}_{L}\right|\left|\Psi_{i}>\right|^{2}=\left|\left\langle\psi_{f}\left|\sum_{j=1}^{N+1} r_{j}\right| \psi_{i}\right\rangle\right|^{2},
$$


where $\Psi_{i}$ and $\Psi_{f}$ are the initial and final state wave functions, and $\mathbf{D}_{L}$ is the dipole operator in length form.

\section{Computations}

BPRM computations are carried out using the R-matrix package of codes $[13,14,8]$ for various stages. They are initiated with the wave function of the core as the initial input. The core wave function for P III was obtained from atomic structure calculations using code SUPERSTRUCTURE (SS) [15, 16]. SS uses Thomas-Fermi-Dirac-Amaldi potential and includes relativistic contributions in Breit-Pauli approximation. Table I presents ground and 17 excited fine structure levels of P III included in the wave function expansion of P II. They were obtained from optimization of 19 configurations up to $5 \mathrm{~s}$ orbital of P III, $3 s^{2} 3 p(1), 3 s 3 p^{2}(2), 3 s^{2} 3 d(3), 3 s^{2} 4 s(4), 3 s^{2} 4 p(5), 3 p^{3}(6), 3 s 3 p 3 d(7)$, $3 s^{2} 4 d(8), 3 s^{2} 4 f(9), 3 s^{2} 5 s(10), 3 s 3 p 4 s(11), 3 s 3 p 4 p(12), 3 s 3 p 4 d(13), 3 s 3 p 4 f(14)$, $3 p^{2} 3 d(15), 3 p^{2} 4 s(16), 3 p^{2} 4 p(17), 3 p^{2} 4 d(18), 3 p^{2} 4 f(19)$. The calculated energies from SS are compared with observed values (listed at NIST[18] from compilation by Martin et al. [17]) in the table. Comparison shows agreement between SS and observed values within a few percent mostly. For accurate energy positions of the resonances the calculated core P III were replaced by the observed energies listed at NIST [18] website, during diagonalization of the $(\mathrm{N}+1)$-electron Hamiltonian.

The wave function of $\mathrm{P}$ II included $0 \leq \ell \leq 10$ partial waves for the interacting electron and 16 continuum functions for the R-matrix basis sets. The R-matrix boundary was chosen to be large enough, $15 a_{o}$, to accommodate the bound orbitals. The second term of the wave function, which represents the bound state correlation functions, included $39(N+1)$-particle configurations with orbital occupancies from minimum to a maximum number as given within parentheses of the orbitals $1 \mathrm{~s}(2-2), 2 \mathrm{~s}(2-2), 2 \mathrm{p}(6-6), 3 \mathrm{~s}(0-2), 3 \mathrm{p}(0-4), 3 \mathrm{~d}(0-3), 4 \mathrm{~s}(0-2), 4 \mathrm{p}(0-$ $2), 4 \mathrm{~d}(0-2), 4 \mathrm{f}(0-1), 5 \mathrm{~s}(0-1))$.

Photoionization cross sections are obtained with consideration of radiation damping for all bound levels using the BPRM $R$-matrix codes $[13,8]$. The narrow resonances of photoionization were delineated at a very fine energy mesh.

\section{Results and Discussions}

Detailed study of photoionization cross sections $\left(\sigma_{P I}\right)$ of Si-like phosphorus ion, P II, are presented. As a basic element of evolution of life, it is important to study the features to help in identifying this element in astrophysical and laboratory plasmas. A total of 475 bound levels of P II with $n \leq 10,0 \leq l \leq$ $9,0 \leq J \leq 7$ of even and odd parities were found. They have been identified spectroscopically using the theoretical procedure based on the quantum defect analysis, percentage of channels contribution, and angular momenta algebra [19]. A sample table of energy comparison is given in Table II and the complete table will be available electronically from database NORA-Atomic-Data. Photoionization cross sections of all 475 bound fine structure levels of P II are 
Figure 1: Photoionization cross sections, $\sigma_{P I}$, of the ground ${ }^{3} P_{0}$ (a) and two excited, ${ }^{3} P_{1,2}$ (b,c) levels of the ground configuration $3 s^{2} 3 p^{2}$ of P II. The sharp resonance at the threshold is due to coupling of fine structure channels.

presented. This will enable complete modelings, such as, of plasma opacities, synthetic spectrum, and diagnostics. Examples of $\sigma_{P I}$ illustrating important characteristic features are discussed below.

Photoionization cross sections of the ground, ${ }^{3} P_{0}$ and two excited, ${ }^{3} P_{1,2}$ levels of the ground configuration $3 s^{2} 3 p^{2}$ of $\mathrm{P}$ II are presented in Figure 1. They look similar, except some resonances. as the levels belong to the same state ${ }^{3} P$. Each level shows existence of narrow resonance at the ionization threshold followed by a prominent broad resonant structure. These indicate high rate for ionization at low energy and the inverse process of recombination at low temperature supporting high reactivity of this low charge ion. The broad structure is very much similar to that found in photoionization of ${ }^{3} \mathrm{P}$ ground state of Si I in Figure 1 of [5]). Si I is isoelectronic to P II and the element before $\mathrm{P}$ in the periodic table. However, the narrow resonance appearing at ionization threshold in P II is missing in $\sigma_{P I}$ of Si I. Calculations for Si I were carried out in nonrelativistic LS coupling approximation and these resonances were not allowed in the approximation. The ground state $3 s^{2} 3 p^{2}\left({ }^{3} P\right)$ can photoionize to the three allowed states, ${ }^{3} S^{o},{ }^{3} P^{o},{ }^{3} D^{o}$. The broad structure can be explained as the combination of autoionizing states that belong to the Rydberg series of states $\left.3 s 3 p^{2}\left({ }^{4} P\right) \nu l\right)\left({ }^{3} S^{o},{ }^{3} P^{o},{ }^{3} D^{o}\right)$ converging on to excited core state $3 s 3 p^{2}\left({ }^{4} P\right)$. LS coupling does not allow the excitation of the fine structure level $2 p\left({ }^{2} P_{3 / 2}^{o}\right)$. Inclusion of relativistic effects for P II has allowed channels $2 p\left({ }^{2} P_{3 / 2}^{o}\right) \nu s_{1 / 2}$ and $2 p\left({ }^{2} P_{3 / 2}^{o}\right) \nu d_{3 / 2,5 / 2}$ to form ${ }^{3} S_{1}^{o},{ }^{3} P_{1}^{o},{ }^{3} D_{1}^{o}$ levels, and hence the autoionizing resonance at the threshold. Relativistic effects are not important in general for low charged ions, such as, P II, but can introduce important sharp resonances in the low energy region.

Various features in photoionization of the ground level can be interpreted through the partial photoionization cross sections of the level leaving the residual ion in different core states. Figure 2 presents partial cross sections of ground level ${ }^{3} P_{0}$ of $\mathrm{P}$ II leaving the core ion in the ground $3 s^{2} 3 p\left({ }^{2} P_{1 / 2}^{o}\right)$ and next seven excited $3 s^{2} 3 p\left({ }^{2} P_{3 / 2}^{o}\right), 3 s 3 p^{3}\left({ }^{4} P_{1 / 2,3 / 2,5 / 2},{ }^{2} D_{3 / 2,5 / 2},{ }^{2} S_{1 / 2}\right)$ levels of P III (listed in Table 1). Panel (a) which presents partial $\sigma_{P I}$ for leaving the core at the ground level $3 s^{2} 3 p\left({ }^{2} P_{1 / 2}^{o}\right)$ illustrates that the narrow resonance at threshold belongs to Rydberg series of $3 s^{2} 3 p\left({ }^{2} P_{3 / 2}^{o}\right) \nu l$ where $\nu$ is the effective quantum number. There is no fine structure splitting of ${ }^{2} P^{o}$ in LS coupling and hence no such resonance can be formed. However, this resonance appears naturally in photoionization of P II as reported in [6]. Panels (c,f,g) which present partial $\sigma_{P I}$ leaving the core at states $3 s 3 p^{2}\left({ }^{4} P_{5 / 2},{ }^{2} D_{3 / 2,5 / 2}\right)$ show that the enhanced resonant structure with peaks at about 1.15 Ry of photoelectron energy have appeared as the enhanced background at about 2.65 Ry in the cross sections of ${ }^{3} P_{0,1,2}$ in Figure 1 
Figure 2: Partial photoionization cross sections, $\sigma_{P I}$, of the ground ${ }^{3} P_{0}$ level of $\mathrm{P}$ II leaving the core in the ground (a) and next seven excited levels, $3 s^{2} 3 p\left({ }^{2} P_{3 / 2}^{o}\right)$, $3 s 3 p^{3}\left({ }^{4} P_{1 / 2,3 / 2,5 / 2},{ }^{2} D_{3 / 2,5 / 2},{ }^{2} S_{1 / 2}\right)$ (b-h) of core P III.

Figure 3: Photoionization cross sections, $\sigma_{P I}$, of the three levels of equivalent state, $3 s 3 p^{3}\left({ }^{3} D_{1,2,3}^{o}\right)$ (a-c) of P II. Relativistic fine structure effect is illustrated in producing i) the narrow and sharp resonance at the threshold and ii) enhancing the background cross section at energies 1.3845 and 1.547 Ry (pointed by arrows) due to the allowed dipole transitions of the core ground level $3 s^{2} 3 p\left({ }^{2} P_{1 / 2}^{o}\right)$ to excited levels $3 s 3 p^{2}\left({ }^{4} P_{3 / 2}\right), 3 s 3 p^{2}\left({ }^{2} D_{3 / 2}\right)$. The effects are compared with $\sigma_{P I}$ of isoelectronic element Si I (d) for which there is no threshold resonance due to its no consideration of relativistic effects.

The threshold resonance due to fine structure couplings of channels for the ground level of P II is found to exist in other equivalent states of P II. Figure 3 shows this resonance appearing in $\sigma_{P I}$ of the three levels of equivalent state, $3 s 3 p^{3}\left({ }^{3} D_{1,2,3}^{o}\right)$. This relativistic effect is compared with the non-relativistic LS coupling photoionization cross sections of $3 s 3 p^{3}\left({ }^{3} D^{o}\right)$ state of Si I which is isoelectronic to P II [5]. As in the case of the ground state, $\sigma_{P I}$ of Si I does not show the existence of this resonances since it is not allowed in LS coupling. This resonance should give important contribution in photoabsorption at low temperature plasma. It may be noted that P II shows more resonances compared to Si I. This can be explained by the fine structure splitting of the core states and thus introducing more Rydberg series of autoionizing resonances in relativistic calculations. The other important feature to note in $\sigma_{P I}$ of these levels is the prominent enhancement in the background cross sections at two energies, as pointed by arrows in the plots, the first one at about $1.3845 \mathrm{Ry}$, which is sum of ionization potential $0.864 \mathrm{Ry}$ and core excitation energy 0.521 Ry for level $3 s 3 p^{2}\left({ }^{4} P_{3 / 2}\right)$, and the second one at about $1.547 \mathrm{Ry}$, which is sum of the ionization energy 0.864 Ry and core excitation energy of 0.683 Ry of level $3 s 3 p^{2}\left({ }^{2} D_{3 / 2}\right)$. Both $3 s 3 p^{2}\left({ }^{4} P_{3 / 2}\right)$ and $3 s 3 p^{2}\left({ }^{2} D_{3 / 2}\right)$ are allowed by strong dipole transition from the core ground level $3 s^{2} 3 p\left({ }^{2} P_{1 / 2}^{o}\right)$ causing the enhancement. The high energy $\sigma_{P I}$ of levels of ${ }^{3} D^{o}$ appear to be decaying faster than that of isoelectronic Si I. The possible reason could be the charge of P II which is following the $1 / z^{2}$ rule for the background cross section where $z$ is the charge of the ion.

The other prominent feature in photoionization is the existence of Seaton or photo-excitation-of-core (PEC) resonances (e.g. [9]) in excited levels with a single valence electron. Here the outer electron remains as a spectator while the core absorbs the photon for a dipole allowed transition from the ground level which is then followed by photoionization as the core drops down to ground level. Hence regardless of the ionization potential of the excited level these resonances appear at the same energy positions when the photon absorption matches to that of the core excitation. Figure 4 illustrates these Seaton (who explained it in [20] for the first time) or PEC resonances for photoionization of three excited levels, $3 s^{2} 3 p 6 p\left({ }^{3} P_{0}\right)$ (a), $3 s^{2} 3 p 4 p\left({ }^{3} D_{2}\right)$ (b) and $3 s^{2} 3 p 5 d\left({ }^{3} D_{2}^{o}\right)$ (c) 
Figure 4: Photoionization cross sections, $\sigma_{P I}$, of three excited levels, $3 s^{2} 3 p 6 p\left({ }^{3} P_{0}\right)$ (a), $3 s^{2} 3 p 4 p\left({ }^{3} D_{2}\right)$ (b) and $3 s^{2} 3 p 5 d\left({ }^{3} D_{2}^{o}\right)$ (c) of P II illustrating photo-excitation-of-core (PEC) or Seaton resonances which occur at the excitation energies of the core as pointed out by the arrows. It can be seen that core excitations not only introduced sharp resonances, they enhanced the background by orders of magnitude.

of P II. The figure demonstrates the importance of these resonances, positions are pointed by arrows, for excited levels which are often treated as hydrogenic. The core excitations to dipole allowed levels introduce not only sharp peak, can also enhance the background considerably, by orders of magnitude as can be seen for the four PEC positions around 1 Ry. Table III lists the core excitations for possible formation of Seaton resonances and their energy positions. As the table shows, there are several excited levels around 1 Ry for dipole allowed transitions of the core. It may be noted in the figure that each level is also showing threshold resonance.

\section{Conclusion}

Photoionization of ( $\mathrm{h} \nu+\mathrm{P}$ II $\rightarrow \mathrm{e}+\mathrm{P}$ III) is studied in detail with emphasis on the resonant and background features. Of particular interest is the sharp resonance at the ionization threshold due to relativistic effects, but not possible in LS coupling, for many levels. This may have important impact on the recombination rates, photoionization rates at low temperature. Enhancement has been found at excited core thresholds. The cross sections of excited levels of single valence electrons show dominance of Seaton resonances over the background. The prominent features are expected to contribute considerably in various laboratory and astrophysical models. The present work reports photoionization cross sections of a large set, 475 in total, levels with $\mathrm{n} \leq 10$ and $l \leq 9$ and $0 \leq J \leq 7$ of even and odd parities. Based on the accuracy of the wave function expansion, R-matrix method with inclusion of relativistic effects, and very good agreement with experiment [6], the accuracy of the presented cross sections is estimated to be $15-20 \%$ and the amount of data are expected to provide a complete and precise modeling for various applications.

The complete set of data for photoionization cross sections and the energy levels are available on-line from NORAD-Atomic-Data website at:

http://norad.astronomy.ohio-state.edu/

Acknowledgments

This work was supported partially by NSF AST-1312441 and AST-1109088. The computational work was carried out at the Ohio Supercomputer Center (OSC) in Columbus Ohio. 


\section{References}

[1] Molaro P, Levshakov SA, DOdorico S, Bonifacio P, Centurion M. UVES Observations of QSO 0000-2620: Argon and Phosphorus Abundances in the Dust-free Damped Ly System at zabs $=3.3901$. Astrophys. J. 2001;549:90-99

[2] Welsh BY, Sfeir DM,Sallmen S, Lallement R. Far Ultraviolet Spectroscopic Explorer observations of high-velocity gas associated with the Monoceros Loop SNR. A\&A 2001;372:516-526

[3] The Opacity Project Team .The Opacity Project, Vol 1, 1995, Vol. 2, 1996, Institute of Physics Publishing

[4] Mendoza C, Zeippen CJ .Photoionisation cross sections of the ground states of neutral Si, P and S. J.Phys.B 1988;21:259-266

[5] Nahar SN, Pradhan AK .Atomic Data For Opacity Calculations: XVI. Photoionization and oscillator strengths of $\mathrm{Si}-$ like ions $\mathrm{Si} 0, \mathrm{~S} 2+, \mathrm{Ar} 4+, \mathrm{Ca} 6+$. J. Phys. B 1993;26:1109-1127

[6] Hinojosa G, Hernandez E, Antillon A, et al .Photoionization Cross Sections of P II: Theory \& Measurement. 46th Annual Meeting of the APS Division of Atomic, Molecular and Optical Physics (DAMOP), 2015; Vol 60 No 7: Abstract: T5.00008 No 7, June 8-12; Columbus, Ohio

[7] Hummer DG, Berrington KA, Eissner W, Pradhan AK, Saraph HE, \& Tully JA .Atomic data from the IRON Project. 1: Goals and methods. Astron. Astrophys. 1003;279:298-309

[8] Zhang HL, Nahar SN, Pradhan AK. Close coupling R-matrix calculations for electron-ion recombination cross sections. J. Phys. B 1999;32:1459-1479

[9] Pradhan AK, Nahar SN .Atomic Astrophysics and Spectroscopy (Cambridge University press, 2011)

[10] Burke PG, Robb WD .R-matrix theory of atomic processes. Adv. At. Mol. Phys. 1975;11:143-214

[11] Seaton MJ, J. Phys. B 1987;20:6363-6378

[12] Berrington KA, Burke PG, Butler K, Seaton MJ, Storey PJ, Taylor KT, \& Yan Yu .Atomic data for opacity calculations. II. Computational methods. J. Phys. B 1987;20:6379-6397

[13] Berrington KA, Eissner W, Norrington PH .RMATRIX1: Belfast atomic R-matrix codes. Comput. Phys. Commun. 1995;92: 290-420

[14] Nahar SN \& Pradhan AK .Unified treatment of electron-ion recombination in the close-coupling approximation. Phys. Rev. A 1994;49: 1816-1835 
[15] Eissner W, Jones M, Nussbaumer H, Techniques for the calculation of atomic structures and radiative data including relativistic corrections. Comput. Phys. Commun. 1974;8:270-306

[16] Nahar SN, Eissner W, Chen GX, Pradhan AK. Atomic data from the Iron Project - LIII. Relativistic allowed and forbidden transition probabilities for Fe XVII. A\&A 2003;408:789-801

[17] Martin WC, Zalubas R, Musgrove A. Energy Levels of Phosphorus, P I through P XV, J. Phys. Chem. Ref. Data 14, 751802 (1985)

[18] Website of NIST table of compiled energies http://physics.nist.gov/cgibin/AtData/main_asd

[19] Nahar S N, Pradhan A K .Large-scale Breit-Pauli R-matrix calculations for transition probabilities of Fe V. Phys. Scr. 2000;61:675-689

[20] Yu Y \& Seaton MJ .Atomic data for opacity calculations. IV. Photoionisation cross sections for C II. J. Phys. B 1987;20:6409-6429 


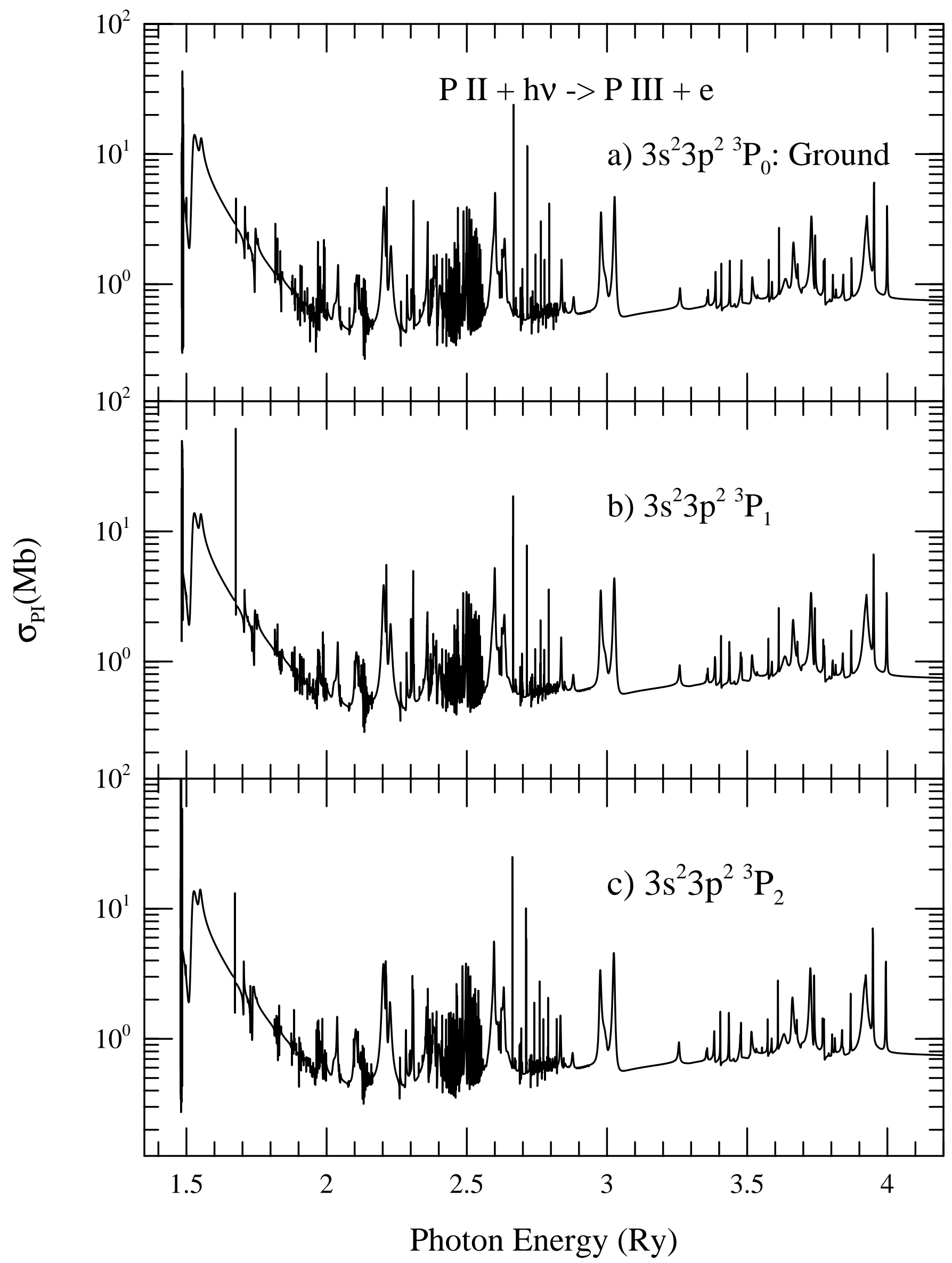




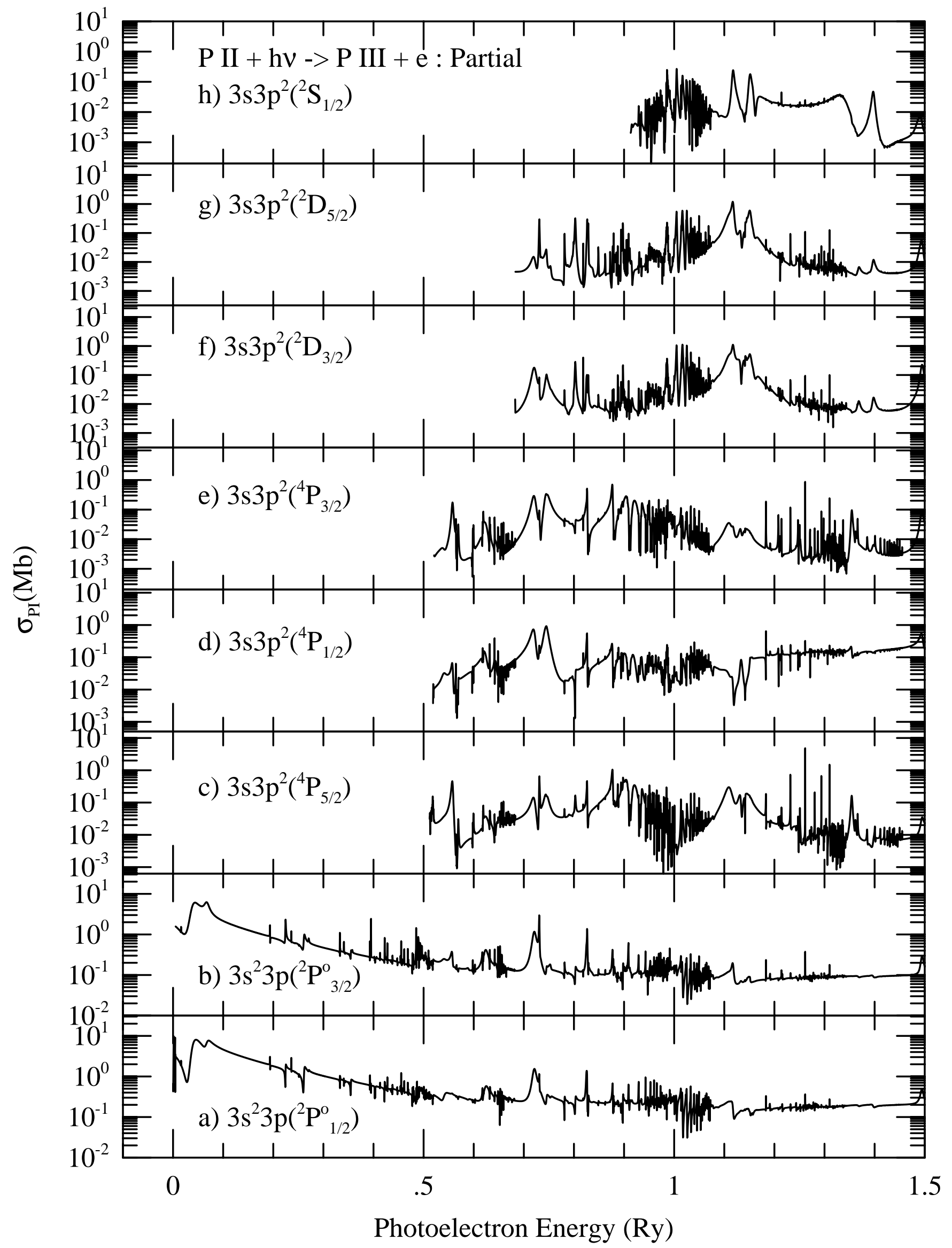




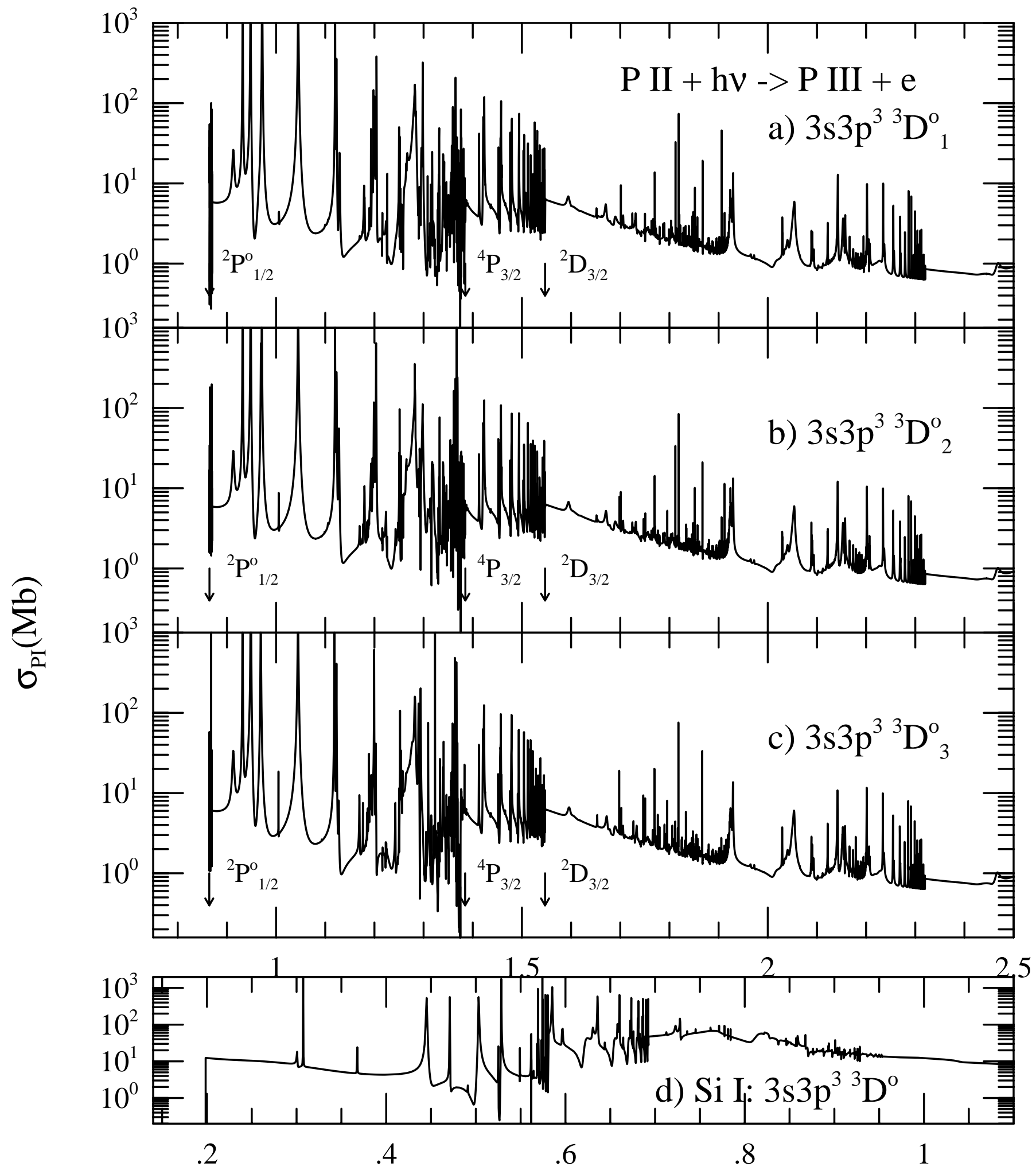

Photon Energy (Ry) 


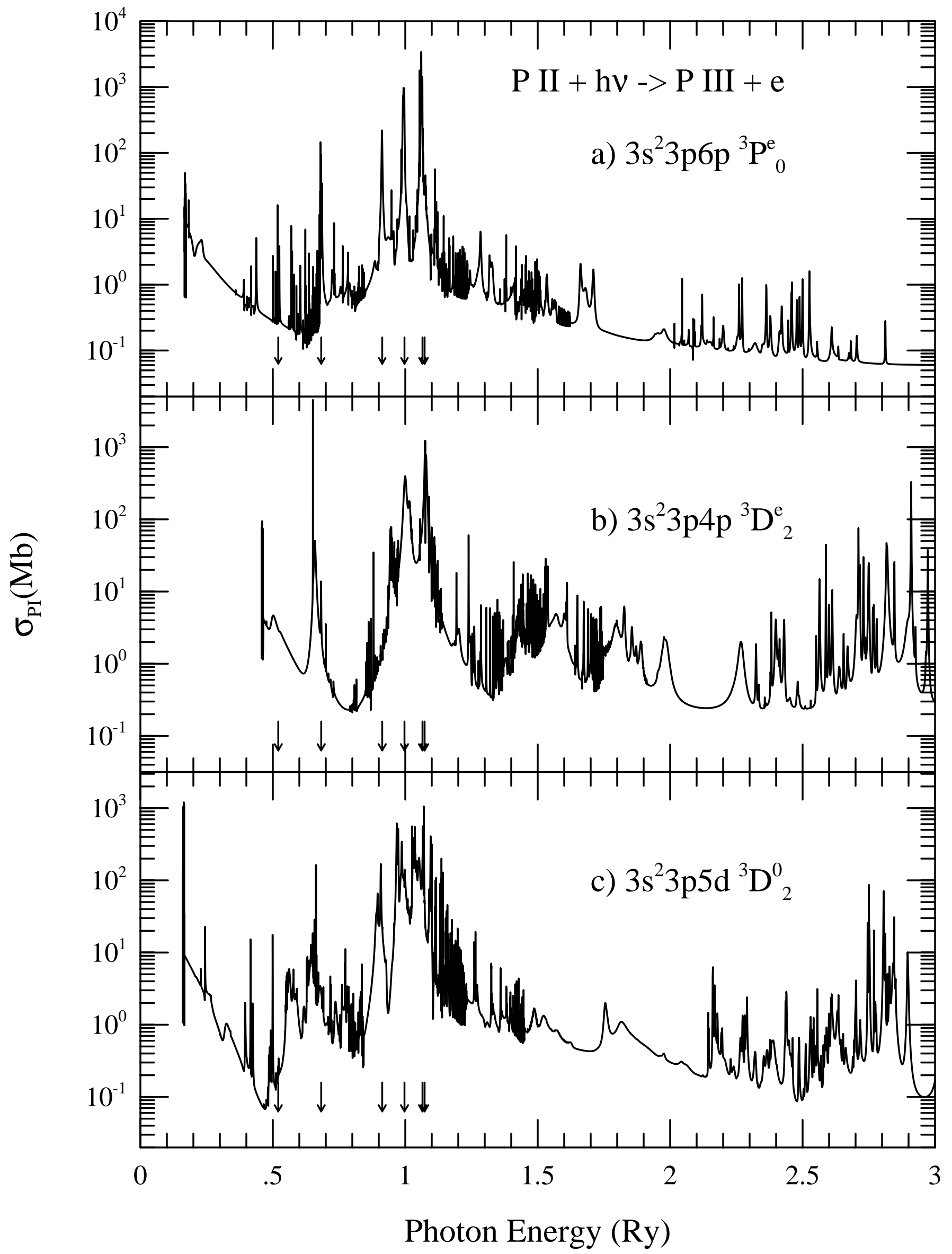


Table 1: Comparison between calculated BPRM and compiled energies by Martin et al [? ] (listed at NIST website [? ]) of P II. $I_{J}$ is the calculated level index for its position in its $J \pi$ symmetry. Negative sign for energies omitted for convenience

\begin{tabular}{llrrr}
\hline \multicolumn{2}{c}{ Level } & $J: I_{J}$ & $E(\mathrm{Ry}, \mathrm{BPRM})$ & $E(\mathrm{Ry}, \mathrm{NIST})$ \\
\hline $3 s 23 p 2$ & ${ }^{3} P^{e}$ & $2: 1$ & $1.4539 \mathrm{E}+00$ & $1.4799 \mathrm{E}+00$ \\
$3 s 23 p 2$ & ${ }^{3} P^{e}$ & $1: 1$ & $1.4566 \mathrm{E}+00$ & $1.4824 \mathrm{E}+00$ \\
$3 s 23 p 2$ & ${ }^{3} P^{e}$ & $0: 1$ & $1.4581 \mathrm{E}+00$ & $1.4838 \mathrm{E}+00$ \\
$3 s 23 p 2$ & ${ }^{1} D^{e}$ & $2: 2$ & $1.3772 \mathrm{E}+00$ & $1.3982 \mathrm{E}+00$ \\
$3 s 23 p 2$ & ${ }^{1} S^{e}$ & $0: 2$ & $1.2615 \mathrm{E}+00$ & $1.2568 \mathrm{E}+00$ \\
$3 s 3 p 3$ & ${ }^{5} S^{o}$ & $2: 1$ & $1.0417 \mathrm{E}+00$ & $1.0601 \mathrm{E}+00$ \\
$3 s 3 p 3$ & ${ }^{3} D^{o}$ & $3: 1$ & $8.6300 \mathrm{E}-01$ & $8.6413 \mathrm{E}-01$ \\
$3 s 3 p 3$ & ${ }^{3} D^{o}$ & $2: 2$ & $8.6332 \mathrm{E}-01$ & $8.6453 \mathrm{E}-01$ \\
$3 s 3 p 3$ & ${ }^{3} D^{o}$ & $1: 1$ & $8.6351 \mathrm{E}-01$ & $8.6477 \mathrm{E}-01$ \\
$3 s 3 p 3$ & ${ }^{3} P^{o}$ & $2: 3$ & $7.5860 \mathrm{E}-01$ & $7.3613 \mathrm{E}-01$ \\
$3 s 3 p 3$ & ${ }^{3} P^{o}$ & $1: 2$ & $7.5816 \mathrm{E}-01$ & $7.3487 \mathrm{E}-01$ \\
$3 s 3 p 3$ & ${ }^{3} P^{o}$ & $0: 1$ & $7.5806 \mathrm{E}-01$ & $7.3469 \mathrm{E}-01$ \\
$3 s 23 p 3 d$ & ${ }^{1} D^{o}$ & $2: 4$ & $7.4998 \mathrm{E}-01$ & $7.3226 \mathrm{E}-01$ \\
$3 s 23 p 4 s$ & ${ }^{3} P^{o}$ & $2: 5$ & $6.6419 \mathrm{E}-01$ & $6.5189 \mathrm{E}-01$ \\
$3 s 23 p 4 s$ & ${ }^{3} P^{o}$ & $1: 3$ & $6.6765 \mathrm{E}-01$ & $6.5504 \mathrm{E}-01$ \\
$3 s 23 p 4 s$ & ${ }^{3} P^{o}$ & $0: 2$ & $6.6899 \mathrm{E}-01$ & $6.5636 \mathrm{E}-01$ \\
$3 s 23 p 3 d$ & ${ }^{3} F^{o}$ & $4: 1$ & $6.5446 \mathrm{E}-01$ & $6.2050 \mathrm{E}-01$ \\
$3 s 23 p 3 d$ & ${ }^{3} F^{o}$ & $3: 2$ & $6.5651 \mathrm{E}-01$ & $6.2265 \mathrm{E}-01$ \\
$3 s 23 p 3 d$ & ${ }^{3} F^{o}$ & $2: 6$ & $6.5799 \mathrm{E}-01$ & $6.2423 \mathrm{E}-01$ \\
$3 s 23 p 4 s$ & ${ }^{1} P^{o}$ & $1: 4$ & $6.4807 \mathrm{E}-01$ & $6.2961 \mathrm{E}-01$ \\
\hline
\end{tabular}


Table 1: Possible dipole allowed transitions from the ground level $3 s^{2} 3 p\left({ }^{2} P_{1 / 2}^{o}\right)$ to various excited levels of core P III for introduction of Seaton or PEC resonances. The level number corresponds to that of Table I.

\begin{tabular}{cccccc}
\hline $\begin{array}{c}\text { Level } \\
\text { Numbers }\end{array}$ & $\begin{array}{c}\text { Core } \\
\text { Transition }\end{array}$ & $\begin{array}{c}\text { E(Ry) } \\
\text { Position }\end{array}$ & $\begin{array}{c}\text { Level } \\
\text { Numbers }\end{array}$ & $\begin{array}{c}\text { Core } \\
\text { Transition }\end{array}$ & $\begin{array}{c}\mathrm{E}(\mathrm{Ry}) \\
\text { Position }\end{array}$ \\
\hline & & & & \\
$1-4$ & $3 s^{2} 3 p\left({ }^{2} P_{1 / 2}^{o}\right)-3 s 3 p^{2}\left({ }^{4} P_{1 / 2}\right)$ & 0.5187 & $1-9$ & $3 s^{2} 3 p\left({ }^{2} P_{1 / 2}^{o}\right)-3 s 3 p^{2}\left({ }^{2} P_{1 / 2}\right)$ & 0.9936 \\
$1-5$ & $3 s^{2} 3 p\left({ }^{2} P_{1 / 2}^{o}\right)-3 s 3 p^{2}\left({ }^{4} P_{3 / 2}\right)$ & 0.5206 & $1-10$ & $3 s^{2} 3 p\left({ }^{2} P_{1 / 2}^{o}\right)-3 s 3 p^{2}\left({ }^{2} P_{3 / 2}\right)$ & 0.9970 \\
$1-6$ & $3 s^{2} 3 p\left({ }^{2} P_{1 / 2}^{o}\right)-3 s 3 p^{2}\left({ }^{2} D_{3 / 2}\right)$ & 0.6827 & $1-11$ & $3 s^{2} 3 p\left({ }^{2} P_{1 / 2}^{o}\right)-3 s^{2} 3 d\left({ }^{2} P_{3 / 2}\right)$ & 1.065 \\
$1-8$ & $3 s^{2} 3 p\left({ }^{2} P_{1 / 2}^{o}\right)-3 s 3 p^{2}\left({ }^{2} S_{1 / 2}\right)$ & 0.9131 & $1-13$ & $3 s^{2} 3 p\left({ }^{2} P_{1 / 2}^{o}\right)-3 s^{2} 4 s\left({ }^{2} S_{1 / 2}\right)$ & 1.073 \\
\hline
\end{tabular}


Table 1: Levels and energies $\left(E_{t}\right)$ of core ion P III included in the wave function expansion of P II. Calculated energies from SUPERSTRUCTURE (SS) are compared with those in the compiled table of NIST [?].

\begin{tabular}{rlrll}
\hline & Level & $J_{t}$ & $\begin{array}{l}E_{t}(\mathrm{Ry}) \\
\mathrm{NIST}\end{array}$ & $\begin{array}{l}E_{t}(\mathrm{Ry}) \\
\mathrm{SS}\end{array}$ \\
\hline 1 & $3 s^{2} 3 p\left({ }^{2} P^{o}\right)$ & $1 / 2$ & 0.0 & 0. \\
2 & $3 s^{2} 3 p\left({ }^{2} P^{o}\right)$ & $3 / 2$ & 0.005095 & 0.00429 \\
3 & $3 s 3 p^{2}\left({ }^{4} P\right)$ & $5 / 2$ & 0.523559 & 0.51278 \\
4 & $3 s 3 p^{2}\left({ }^{4} P\right)$ & $3 / 2$ & 0.520570 & 0.51028 \\
5 & $3 s 3 p^{2}\left({ }^{4} P\right)$ & $1 / 2$ & 0.518708 & 0.50874 \\
6 & $3 s 3 p^{2}\left({ }^{2} D\right)$ & $3 / 2$ & 0.682693 & 0.70551 \\
7 & $3 s 3 p^{2}\left({ }^{2} D\right)$ & $5 / 2$ & 0.682957 & 0.70565 \\
8 & $3 s 3 p^{2}\left({ }^{2} S\right)$ & $1 / 2$ & 0.913094 & 0.99414 \\
9 & $3 s 3 p^{2}\left({ }^{2} P\right)$ & $1 / 2$ & 0.993621 & 1.03335 \\
10 & $3 s 3 p^{2}\left({ }^{2} P\right)$ & $3 / 2$ & 0.997044 & 1.03613 \\
11 & $3 s^{2} 3 d\left({ }^{2} D\right)$ & $3 / 2$ & 1.065039 & 1.14381 \\
12 & $3 s^{2} 3 d\left({ }^{2} D\right)$ & $5 / 2$ & 1.065142 & 1.14392 \\
13 & $3 s^{2} 4 s\left({ }^{2} S\right)$ & $1 / 2$ & 1.073800 & 1.10909 \\
14 & $3 s^{2} 4 p\left({ }^{2} P^{o}\right)$ & $1 / 2$ & 1.28832 & 1.34867 \\
15 & $3 s^{2} 4 p\left({ }^{2} P^{o}\right)$ & $3 / 2$ & 1.28956 & 1.34955 \\
16 & $3 p^{3}\left({ }^{2} D^{o}\right)$ & $3 / 2$ & 1.342508 & 1.38637 \\
17 & $3 p^{3}\left({ }^{2} D^{o}\right)$ & $5 / 2$ & 1.343073 & 1.38678 \\
18 & $3 p^{3}\left({ }^{4} S^{o}\right)$ & $3 / 2$ & 1.455433 & 1.49071 \\
\hline \hline
\end{tabular}

